\title{
Progresso genético e estrutura populacional do rebanho Nelore no Estado da Bahia
}

\author{
Carlos Henrique Mendes Malhado(1), Paulo Luiz Souza Carneiro(1), Derval Gomes Pereira(2) \\ e Raimundo Martins Filho ${ }^{(3)}$
}

\begin{abstract}
(1)Universidade Estadual do Sudoeste da Bahia (UESB), Departamento de Ciências Biológicas, Avenida José Moreira Sobrinho, s/no, Jequiezinho, CEP 45200-000 Jequié, BA. E-mail: carlosmalhado@gmail.com, plscarneiro@gmail.com (2)UESB, Departamento de Química e Exatas. E-mail: derval.gomespereira@gmail.com ${ }^{(3)}$ Universidade para o Desenvolvimento do Estado e da Região do Pantanal, Campus I Miguel Couto, Caixa Postal 2.153, CEP 79003-010 Campo Grande, MS. E-mail: rmartinsfilho@yahoo.com.br
\end{abstract}

Resumo - O objetivo deste trabalho foi avaliar a estrutura populacional e o progresso genético e fenotípico de características de desenvolvimento ponderal, em bovinos da raça Nelore, no Estado da Bahia. Foram utilizadas informações de pedigree de animais nascidos no período de 1955 a 2007, e dados dos pesos ajustados aos 205, 365 e 550 dias de idade, de bovinos nascidos de 1970 a 2006. As estimavas dos coeficientes de herdabilidade foram de moderadas a altas, quanto aos pesos ajustados nas três idades. Os rebanhos apresentaram ganho genético positivo nas três características, porém, de baixa magnitude. A mudança fenotípica no decorrer dos anos foi quase exclusivamente relacionada à melhoria ambiental. O tamanho efetivo da população de Nelore do Estado da Bahia tem sido alto em alguns períodos, o que tem levado a menor incremento de endogamia e maiores ganhos genéticos. O intervalo de geração é alto e sua redução é importante para que se possa alcançar maior ganho genético anual.

Termos para indexação: endogamia, intervalo de geração, ganho genético, pedigree, seleção, tamanho efetivo.

\section{Genetic progress and population structure in Nellore cattle in Bahia State, Brazil}

\begin{abstract}
The objective of this work was to evaluate the population structure as well as the genetic and phenotypic progress in growth development traits of Nellore cattle raised in Bahia state, Brazil. Pedigree data of bovines, born between 1955 and 2007, and weight values adjusted to 205, 365, and 550 days of age, from 1970 to 2006, were used. The heritability coefficients were moderate to high for the adjusted weights, in all three ages. The herds presented a positive but small genetic gain. The phenotypic changes over years were almost exclusively related to environmental improvements. The effective size of the Nellore population raised in Bahia state has increased over some periods, thereby decreasing the inbreeding rate and leading to higher genetic gains. The generation interval is high and its reduction is important, in order to increase the annual genetic gain.
\end{abstract}

Index terms: inbreeding, generation interval, genetic gain, pedigree, selection, effective size.

\section{Introdução}

Informações de pedigree têm sido muito utilizadas para avaliar a diversidade genética e a estrutura populacional, em diversas raças de animais domésticos (Faria et al., 2001, 2002; Vercesi Filho et al., 2002). Os estudos da estrutura das populações, mediante informações de pedigree, podem clarear importantes circunstâncias que afetam o histórico genético das populações (Valera et al., 2005). Adicionalmente, alguns parâmetros populacionais, largamente dependentes do manejo e dos sistemas de acasalamento, têm grande impacto na variabilidade genética.
Atualmente, têm sido desenvolvidas pesquisas com modernos métodos de avaliação genética e de estimação de parâmetros genéticos, para diferentes critérios de seleção; outro enfoque aborda somente as estruturas das populações. Todos são extremamente importantes no melhoramento genético. Entretanto, poucos estudos abordam as possíveis inter-relações entre a estrutura populacional e o progresso genético.

De acordo com Malhado et al. (2008), para a viabilidade dos programas de melhoramento genético, é imprescindível que se conheçam os diferentes fatores que interferem potencialmente na seleção e 
no progresso genético, como por exemplo, tamanho efetivo, intervalo de gerações e variabilidade genética.

Muir (2000) relata que, entre os principais fatores que influem na resposta à seleção, além do tamanho efetivo da população estão a acurácia e a intensidade de seleção. Estudos indicam que a resposta ótima à seleção pode ser obtida por meio da otimização desses fatores; entretanto, nem todos podem ser otimizados simultaneamente. $\mathrm{O}$ aumento na intensidade de seleção, por exemplo, provoca decréscimo no tamanho efetivo da população e, conseqüentemente, na resposta à seleção.

Meuwissen \& Woolliams (1994) ressaltaram que novas metodologias têm sido desenvolvidas no melhoramento animal como forma de aumentar o ganho genético, como: o BLUP (best linear unbiased prediction) e os núcleos MOET (multiple ovulation and embryo transfer). Entretanto, para esses métodos, as taxas de endogamia são aumentadas concomitantemente, de modo mais intenso em pequenas populações, como no caso de núcleos MOET e de pequenos núcleos para conservação genética. Segundo Muir (2000), o primeiro impacto da endogamia é a perda de alelos, decorrente da oscilação genética, que é diretamente proporcional aos níveis de endogamia.

Alguns estudos com raças zebuínas têm evidenciado pequenos progressos genéticos nas características de desenvolvimento ponderal no Brasil (Oliveira et al., 1995; Euclides Filho et al., 2000; Ferraz Filho et al., 2002), e particularmente no Nordeste (Malhado et al., 2005; Santos et al., 2007).

O monitoramento do progresso genético deve ser preocupação em relação a toda e qualquer raça (Euclides Filho et al., 2000). Estudos abrangentes são imprescindíveis para o desenvolvimento da bovinocultura de uma região, e o conhecimento da evolução genética de uma população é importante para se avaliar o resultado do programa de seleção adotado; além disso a estrutura populacional deve servir como elemento norteador de ações futuras nos programas de melhoramento genético.

O objetivo deste trabalho foi avaliar a inter-relação da estrutura populacional com o progresso genético das características de desenvolvimento ponderal, em bovinos da raça Nelore, no Estado da Bahia.

\section{Material e Métodos}

Os dados utilizados foram provenientes do controle de desenvolvimento ponderal da raça Nelore, da
Associação Brasileira dos Criadores de Zebu. Foram utilizadas informações de pedigree de bovinos nascidos no período de 1950 a 2007 e dados dos pesos ajustados aos 205 (P205), 365 (P365) e 550 (P550) dias de idade, de animais nascidos de 1970 a 2006, no Estado da Bahia. Na Tabela 1, está apresentada a estrutura do conjunto de dados utilizados das três características.

Para obtenção das estimativas das variâncias e dos valores genéticos, empregou-se a metodologia da máxima verossimilhança restrita livre de derivada, por meio de modelos animais unicaracterística, com uso do aplicativo Multiple Traits DerivativeFree Restrict Maximum Likelihood (Boldman et al., 1995). Para P205 e P365, foram utilizados modelos que incluíram os efeitos aleatórios genéticos aditivos (direto e materno), efeito de ambiente permanente materno, a co-variável idade da vaca ao parto (efeitos linear e quadrático), além do efeito fixo de grupo de contemporâneos, tendo-se admitido a co-variância entre os efeitos genéticos direto e materno como nula. Para P550, foram considerados o mesmo efeito fixo e co-variável, porém, apenas o efeito genético aditivo direto foi considerado como aleatório.

Os grupos de contemporâneos constituíram-se de animais do mesmo sexo, época (estação e ano de nascimento), fazenda e regime alimentar. Grupos de contemporâneos, com menos de três animais, foram excluídos das análises.

As estimativas das tendências genéticas e fenotípicas das características foram obtidas pela regressão linear da média da variável dependente (valores genéticos e pesos observados) sobre o ano de nascimento, por meio do SAS (SAS Institute, 1999).

Utilizou-se a regressão não-paramétrica por meio de polinômios articulados ("spline") para descrever as tendências observadas nos gráficos.

Tabela 1. Estrutura de dados no arquivo analisado, relativa aos pesos aos 205 (P205), 365 (P365) e 550 (P550) dias de idade, em bovinos da raça Nelore, no Estado da Bahia.

\begin{tabular}{lrrr}
\hline Estrutura dos dados & P205 & \multicolumn{1}{c}{ P365 } & \multicolumn{1}{c}{ P550 } \\
\hline Pesagens totais $\left(\mathrm{n}^{\mathrm{o}}\right)$ & 29.199 & 18.935 & 13.660 \\
Pesagens utilizadas $\left(\mathrm{n}^{\mathrm{o}}\right)$ & 19.761 & 11.279 & 8.090 \\
Animais na matriz de parentesco $\left(\mathrm{n}^{\mathrm{o}}\right)$ & 29.607 & 17.692 & 12.934 \\
Grupos de contemporâneos $\left(\mathrm{n}^{\mathrm{o}}\right)$ & 1.403 & 1.156 & 967 \\
Touros $\left(\mathrm{n}^{\mathrm{o}}\right)$ & 863 & 665 & 552 \\
Fazendas $\left(\mathrm{n}^{\mathrm{o}}\right)$ & 85 & 72 & 65 \\
\hline
\end{tabular}


O estimador "spline" tem a forma:

$\mathrm{S}(\lambda)=\frac{1}{3} \sum_{\mathrm{i}=1}^{\mathrm{n}}\left(\mathrm{y}_{\mathrm{i}}-\hat{\mathrm{f}}_{\lambda}(x)\right)^{2}+\lambda \int_{-\infty}^{\infty}\left(\hat{\mathrm{f}}_{\lambda}(\mathrm{x})\right)^{2} \mathrm{dx}$ em que: $\hat{f}_{\lambda}$ pertence ao conjunto das funções continuamente diferenciadas, com derivadas de segunda ordem de integráveis quadradas; $\lambda$ é uma constante positiva; $S(\lambda)$ é o estimador de alisamento "spline", que ajusta um polinômio cúbico a cada intervalo entre pontos.

$\mathrm{O} \lambda$ é o parâmetro de alisamento que controla a troca entre a bondade e o alisamento do ajuste. Selecionase um parâmetro de alisamento $\lambda$ por especificar uma constante c na fórmula $\lambda-(\mathrm{Q} / 10) 3 \mathrm{c}$, em que Q é o intervalo interquartílico. Esta fórmula faz com que $\mathrm{c}$ seja independente da unidade de $\mathrm{x}$.

O programa ENDOG (Gutiérrez \& Goyache, 2005) foi utilizado para a análise do pedigree e a estimação dos parâmetros baseados na probabilidade de origem do gene, tamanho efetivo e intervalo de gerações. $\mathrm{O}$ tamanho efetivo da população $(\mathrm{Ne})$ foi estimado para sete períodos de oito anos cada, de 1955 a 2001, por meio da taxa de variação dos coeficientes médios de endogamia entre gerações sucessivas, conforme a fórmula (Falconer \& Mackay, 1996):

$\Delta \mathrm{F}=\frac{\mathrm{F}_{\mathrm{t}}-\mathrm{F}_{\mathrm{t}-1}}{1-\mathrm{F}_{\mathrm{t}-1}}$, com rearranjos $\mathrm{N}_{\mathrm{e}}=\frac{1}{2 \Delta \mathrm{F}}$, em que:

$F_{t}$ é o coeficiente médio de endogamia, estimado na geração atual; $\mathrm{e}_{\mathrm{t}-1}$ é o coeficiente médio de endogamia estimado na geração anterior.

$\mathrm{O}$ número efetivo de fundadores representa o número de animais com igual contribuição, que produziria a mesma variabilidade genética encontrada na população estudada. O número efetivo de ancestrais representa o número mínimo de animais (fundadores ou não) necessário para se explicar a total diversidade genética da população estudada (Gutiérrez \& Goyache, 2005).
Para a classificação do tipo de rebanho (núcleo, multiplicador, comercial e isolado) foram considerados apenas rebanhos com pelo menos 100 animais.

As estatísticas F de Wright (1938) $-\mathrm{F}_{\mathrm{it}}, \mathrm{F}_{\mathrm{st}}$ e $\mathrm{F}_{\text {is }}-$ foram calculadas por meio do programa ENDOG (Gutiérrez \& Goyache, 2005), em que: $F_{\text {it }}$ é o coeficiente médio de endogamia da população; $F_{\text {st }}$ designa o coeficiente médio de endogamia esperado, estimado em populações hipotéticas produzidas pelo acasalamento ao acaso dos pais, dentro de cada período; e $\mathrm{F}_{\text {is }}$ expressa o desvio da casualidade obtida nos acasalamentos atuais. Se $\mathrm{F}_{\text {is }}$ for maior do que zero, então a endogamia real ultrapassa o nível esperado sob acasalamentos ao acaso, e isto implica que os acasalamentos estão acontecendo entre animais mais aparentados do que a média e, conseqüentemente, a população estará dividida em subpopulações. Quando $\mathrm{F}_{\text {is }}$ é menor do que zero, ou a endogamia é evitada ou predomina o acasalamento entre subpopulações.

\section{Resultados e Discussão}

As médias e os desvios-padrão para P205, P365 e P550 foram $167 \pm 34,5,225,9 \pm 49,5$ e $295,8 \pm 71,5 \mathrm{~kg}$, respectivamente.

Os componentes de variância estão apresentados na Tabela 2. Os coeficientes de herdabilidades (efeito genético direto) foram $0,16 \pm 0,02, \quad 0,24 \pm 0,02$ e $0,33 \pm 0,02$ para P205, P365 e P550, respectivamente. Lôbo et al. (2000), com base em 78 trabalhos sobre bovinos nos trópicos, observaram herdabilidade média de 0,30, 0,37 e 0,35 para o efeito direto de P205, P365 e P550, respectivamente. Gianotti et al. (2005) revisaram 331 trabalhos com bovinos e relataram herdabilidade de $0,23,0,27$ e 0,31, para P205, P365 e P550, respectivamente. Lira et al. (2008), em 67 trabalhos com zebuínos, encontraram estimativas médias de herdabilidade direta de 0,28, 0,31 e 0,35, para P205, P365 e P550, respectivamente.

Tabela 2. Estimativas dos componentes de variância e herdabilidade, relativas aos pesos aos 205 (P205), 365 (P365) e 550 (P550) dias de idade, em bovinos da raça Nelore, no Estado da Bahia ${ }^{(1)}$.

\begin{tabular}{lcccrrrr}
\hline Pesos ajustados & $\sigma_{\mathrm{a}}{ }^{2}$ & $\sigma_{\mathrm{m}}{ }^{2}$ & $\sigma_{\mathrm{ep}}{ }^{2}$ & $\sigma_{\mathrm{e}}{ }^{2}$ & $\sigma_{\mathrm{p}}{ }^{2}$ & $\mathrm{~h}_{\mathrm{d}}{ }^{2}$ \\
\hline P205 & 73,91 & 29,06 & 64,06 & 290,78 & 457,82 & $0,16 \pm 0,02$ \\
P365 & 179,62 & 59,27 & 34,43 & 489,88 & $0,06 \pm 0,02$ \\
P550 & 510,69 & - & - & $1.050,10$ & $1.560,80$ & $0,24 \pm 0,02$ & $0,08 \pm 0,02$ \\
\hline
\end{tabular}

${ }^{(1)} \sigma_{\mathrm{a}}{ }^{2}$ é o componente de variância genética aditiva direta; $\sigma_{\mathrm{m}}{ }^{2}$ é o componente de variância genética aditiva materna; $\sigma_{\mathrm{ep}}{ }^{2}$ é a variância de ambiente permanente materno; $\sigma_{\mathrm{e}}^{2}$ é a variância residual; $\sigma_{\mathrm{p}}{ }^{2}$ é a variância fenotípica; $\mathrm{h}_{\mathrm{d}}{ }^{2}$ é a herdabilidade direta; $\mathrm{h}_{\mathrm{m}}{ }^{2}$ é a herdabilidade materna. 
Os coeficientes de herdabilidade genética materna para P205 e P365 foram $0,06 \pm 0,02$ e $0,08 \pm 0,02$, respectivamente. Lira et al.(2008) relataram médias de 0,12 e 0,10 para P205 e P365, respectivamente.

Os valores dos coeficientes de herdabilidade, para os efeitos genéticos diretos, estimados para as três características, são de média magnitude e indicam variabilidade genética aditiva direta, o que torna evidente a oportunidade de ganho genético por meio da seleção. No entanto, os coeficientes de herdabilidade materna são baixos, o que indica maior dificuldade de seleção direta para esta característica.

As estimativas do intervalo de gerações, para as quatro passagens gaméticas, foram $9,1 \pm 4,6$ (pai-filho),

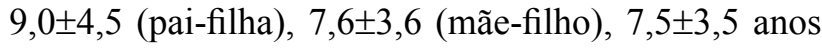
(mãe-filha). $O$ intervalo de gerações médio foi $8,3 \pm 4,2$ anos. Os valores para as passagens gaméticas pai-filho e pai-filha são considerados altos, quando comparados aos intervalos relatados por Vercesi Filho et al. (2002) e Faria et al. (2002), que estimaram médias de 7,2 e 7,1 (pai-filho), 7,2 e 7,2 (pai-filha), 7,0 e 7,2 (mãe-filho) e 6,9 e 7,1 (mãe-filha), para animais registrados das raças Tabapuã e Nelore Mocho, respectivamente.

A otimização do intervalo médio de gerações é de fundamental importância em programas de melhoramento genético, pois intervalos muito grandes diminuem o ganho genético anual quanto às características selecionadas, o que leva a menor retorno econômico do programa (Faria et al., 2001). Autilização de jovens touros avaliados é uma ferramenta para a redução deste intervalo, principalmente, do intervalo pai-filho e pai-filha.

$\mathrm{Na}$ Tabela 3, estão apresentados os números de reprodutores, as médias de filhos por reprodutor e o tamanho efetivo da população, no período de 1955 a 2001, em intervalos de 8 anos. Observa-se que no período de 1963 a 1983, apesar do número de touros ter aumentando três vezes e de o número de vacas aproximadamente seis vezes, o acréscimo no tamanho efetivo foi de apenas $25 \%$. A principal explicação para o pequeno aumento do tamanho efetivo é a utilização intensiva de alguns reprodutores, o que corrobora o fato de a maior média de filhos por reprodutor ter ocorrido no período de 1969 e 1983.

A partir do ano de 1983, ocorreu um aumento acentuado do tamanho efetivo, que atingiu o valor máximo $(469,58)$ no último período (1994-2001). Os períodos de maiores ganhos genéticos, ao longo dos 36 anos estudados, foram de 1983 a 1990 e a partir de 1994 (Figura 1), períodos esses em que o tamanho efetivo da população foi maior. Nos períodos em que os tamanhos efetivos foram menores, os ganhos genéticos foram modestos. Carneiro et al. (2006) afirmam que, ao se utilizarem populações com pequenos tamanhos efetivos para seleção, os resultados podem ser influenciados pela oscilação genética e podem resultar em grandes variações nos ganhos genéticos.

A intensidade de seleção e o tamanho efetivo são fatores antagônicos, ou seja, quando se pratica alta intensidade de seleção, reduz-se o tamanho efetivo. É importante associar a estes dois fatores o coeficiente de herdabilidade e trabalhar no sentido de se aumentar o coeficiente de herdabilidade, pela diminuição da variância ambiental. Os benefícios do aumento da herdabilidade são evidentes pois, com maior precisão na seleção, a adoção de alta intensidade de seleção pouco deverá contribuir para a perda aleatória de alelos favoráveis.

Malhado et al. (2008) frisaram que, para aumento nos ganhos genéticos, são necessários a redução do intervalo de geração, o aumento do tamanho efetivo, o uso de reprodutores avaliados e o controle dos acasalamentos de animais aparentados.

Goddard \& Smith (1990) sugeriram tamanho efetivo mínimo de 40 animais por geração, para maximizar o retorno econômico, enquanto Meuwissen $\&$ Woollians (1994) recomendaram valores entre 31 e

Tabela 3. Número de reprodutores, média de filhos por reprodutor e tamanho efetivo, em intervalos de 8 anos, de 1955 a 2001 , em bovinos da raça Nelore, no Estado da Bahia.

\begin{tabular}{|c|c|c|c|c|c|}
\hline Período & Touros & Média de filhos por ano & Vacas & Média de filhos por ano & Tamanho efetivo \\
\hline $1955-1962$ & 5 & 5,6 & 9 & 1,1 & 3,59 \\
\hline $1962-1969$ & 63 & 8,1 & 251 & 1,2 & 49,90 \\
\hline 1969-1976 & 161 & 11,2 & 972 & 1,3 & 62,81 \\
\hline $1976-1983$ & 195 & 10,8 & 1.505 & 1,4 & 62,43 \\
\hline $1983-1990$ & 244 & 8,3 & 1.815 & 1,4 & 355,92 \\
\hline 1990-1997 & 290 & 7,4 & 1.666 & 1,3 & 458,24 \\
\hline 1994-2001 & 210 & 5,2 & 1.100 & 1,2 & 469,58 \\
\hline
\end{tabular}


250, para prevenir decréscimos no valor adaptativo. Frankham (1995) sugere um tamanho efetivo de 500 animais, para manutenção do potencial evolutivo indefinidamente, contudo, esse autor frisou que, para a prevenção de depressão endogâmica, um tamanho de 50 é suficiente.

O coeficiente de endogamia média aumentou de $0,8 \%$, na segunda geração, para $1,57 \%$, na sétima, contudo, a média de $\mathrm{F}$ para os animais endogâmicos diminuiu de $16,76 \%$, na segunda geração, para $3,85 \%$, na sétima. Esses resultados evidenciam que a endogamia nos rebanhos é baixa, fato explicado pelo aumento do tamanho efetivo nos últimos períodos.
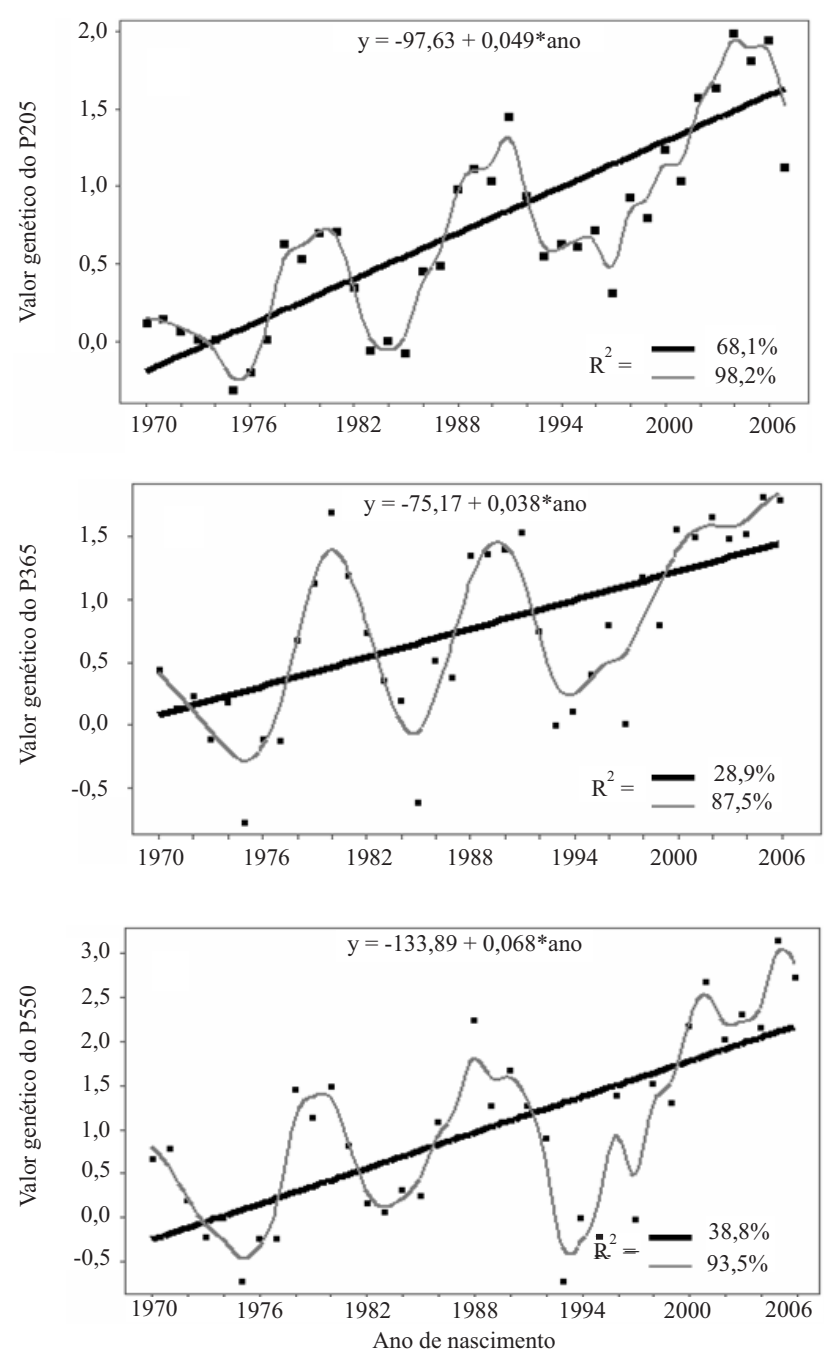

Figura 1. Tendência genética relativa ao efeito direto das características P205, P365 e P550, em bovinos da raça Nelore, no Estado da Bahia.
A contribuição genética dos 10,50 e 414 ancestrais mais influentes explica, respectivamente, 12,5, 25 e $50 \%$ da variabilidade genética de toda a população. $\mathrm{O}$ número efetivo de fundadores foi de 786 , e a endogamia esperada pelo uso desbalanceado dos fundadores foi de $0,06 \%$.

As estatísticas $\mathrm{F}$ de Wright, para a população como um todo, foram dadas por $\mathrm{F}_{\mathrm{is}}, \mathrm{F}_{\mathrm{st}}$ e $\mathrm{F}_{\mathrm{it}}$, cujas estimativas foram -0,0063, 0,0065 e 0,0002, respectivamente. $\mathrm{OF}_{\text {is }}$, que mede o desvio da casualidade nos acasalamentos, foi negativo e próximo a zero e revelou um pequeno predomínio de acasalamentos entre subpopulações, o que contribuiu para minimizar a endogamia. Tal valor negativo está, provavelmente, associado à utilização de inseminação artificial e intercâmbio de reprodutores, práticas importantes para o melhoramento genético da raça e conectabilidade entre os rebanhos.

$\mathrm{O}$ baixo valor de $\mathrm{F}_{\text {st }}$ indica, também, a ausência de estruturação da população, decorrente do fato de que $100 \%$ das fazendas analisadas utilizam reprodutores externos ou inseminação artificial, e que aproximadamente $86 \%$ delas vendem reprodutores. A estruturação pode ser obtida por meio de subpopulações com pequena migração e fluxo gênico entre elas, até subpopulações totalmente isoladas.

Para P205, a tendência genética (regressão linear) do efeito genético direto foi significativa $(p<0,001)$ e igual a $0,049 \mathrm{~kg}$ por ano, o que representa progresso genético de 1,76 kg, nos 36 anos analisados (Figura 1). A tendência genética materna (regressão linear) também foi significativa $(\mathrm{p}>0,05)$ e igual a $0,005 \mathrm{~kg}$ por ano.

As tendências genéticas (regressão linear) para P365 e P550, do efeito genético direto, foram significativas $(p<0,001)$ e iguais a 0,038 e $0,068 \mathrm{~kg}$ por ano, respectivamente, o que equivale ao progresso genético de 1,37 e $2,44 \mathrm{~kg}$, no período estudado de 37 anos (Figura 1). A tendência genética materna para a característica P365 não foi significativa $(p>0,05)$.

Em concordância com esses resultados, Biffani et al. (1999) frisaram que, nas condições de criação típicas do Nordeste, os animais são selecionados pelos criadores mais em razão das características anatômicas e raciais do que pelo desempenho produtivo. Entretanto, esta tendência vem diminuindo nos últimos anos, conforme se pode verificar na Figura 1, em que se pode visualizar um progresso genético crescente e aproximadamente linear, a partir de 1994. Este resultado é confirmado no estudo de Malhado et al. (2005), que relataram 
existência de pequeno ganho genético, nos últimos dez anos, nas características relacionadas à velocidade de crescimento, em bovinos da raça Nelore, em toda região Nordeste.

Os ganhos genéticos no período correspondem aos incrementos de 1,02, 0,60 e 0,82\% em P205, P365 e $\mathrm{P} 550$, respectivamente. Considerando-se a intensidade de seleção de 1,24 (ou seja, retenção de $5 \%$ dos machos e $75 \%$ das fêmeas), as herdabilidades diretas, os desvios-padrão fenotípicos de P205, P365 e P550 e o intervalo de geração dos rebanhos ( 8,3 anos), o progresso genético esperado $(\Delta \mathrm{G})$ seria de $0,82,1,77$ e $3,52 \mathrm{~kg}$ por ano, o que corresponderia a $0,49,0,69$ e $1,19 \%$ da média da população, respectivamente, para P205, P365 e P550. É importante frisar que, se o intervalo de geração fosse reduzido pela metade (aproximadamente 4,15 anos), os possíveis ganhos genéticos por ano seriam duplicados.

As tendências fenotípicas (regressão linear) para os pesos aos 205, 365 e 550 dias de idade, foram significativas $(p<0,001)$ e de $1,40,1,65$ e $2,11 \mathrm{~kg}$ por ano, respectivamente. Pode-se constatar, pelos resultados, que as características estão melhorando com o decorrer dos anos e que os produtores estão conseguindo ganhos fenotípicos anuais significativos no crescimento dos animais. Entretanto, esse progresso deve ser atribuído, principalmente, ao ambiente, visto que a contribuição genética, apesar de positiva, foi baixa. Portanto, este ganho fenotípico tende a atingir o seu limite, se não houver seleção para as características avaliadas neste trabalho, nos próximos anos.

\section{Conclusões}

1. Há possibilidade de ganho genético por meio de seleção, nos rebanhos avaliados, contudo, o progresso genético é pequeno.

2. Os ganhos fenotípicos observados neste trabalho devem ser atribuídos, principalmente, a melhorias no ambiente.

3. O elevado tamanho efetivo da população, observado em alguns períodos, tem resultado em menor incremento de endogamia e em maiores ganhos genéticos, nesses períodos.

4. A inter-relação da estrutura populacional com progresso genético pode servir para nortear ações futuras, que permitirão estratégias para o progresso genético da raça na região.

\section{Agradecimentos}

À Universidade Estadual do Sudoeste da Bahia e à Fundação de Amparo à Pesquisa do Estado da Bahia, pelo apoio financeiro.

\section{Referências}

BIFFANI, S.; MARTINS FILHO, R.; GIORGETTI, A.; BOZZI, R.; LIMA, F.A.M. Fatores ambientais e genéticos sobre o crescimento ao ano e ao sobreano de bovinos Nelore, criados no Nordeste do Brasil. Revista Brasileira de Zootecnia, v.28, p.468-473, 1999.

BOLDMAN, K.G.; KRIESE, L.A.; VAN VLECK, L.D.; VAN TASSEL, C.P.V.; KACHMAN, S.D. A manual for use of MTDFREML: a set of programs to obtain estimates of variances and covariances [DRAFT]. Lincoln: Agricultural Research Service, 1995. 120p.

CARNEIRO, P.L.S.; MALHADO, C.H.M.; EUCLYDES, R.F.; TORRES, R. de A.; LOPES, P.S.; CARNEIRO, A.P.S.; CUNHA, E.E. Oscilação genética em populações submetidas a métodos de seleção tradicionais e associados a marcadores moleculares. Revista Brasileira de Zootecnia, v.35, p.84-91, 2006.

EUCLIDES FILHO, K.; SILVA, L.O.C. da; ALVES, R.G. de O.; FIGUEIREDO, G.R. de. Tendência genética na raça Gir. Pesquisa Agropecuária Brasileira, v.35, p.787-791, 2000.

FALCONER, D.S.; MACKAY, T.F.C. Introduction to quantitative genetics. $4^{\text {th }}$ ed. Harlow: Longman, 1996. 464p.

FARIA, F.J.C.; VERCESI FILHO, A.E.; MADALENA, F.E.; JOSAHKIAN, L.A. Estrutura populacional da raça Nelore Mocho. Arquivo Brasileiro de Medicina Veterinária e Zootecnia, v.54, p.501-509, 2002.

FARIA, F.J.C.; VERCESI FILHO, A.E.; MADALENA, F.E.; JOSAHKIAN, L.A. Parâmetros populacionais do rebanho Gir Mocho registrado no Brasil. Revista Brasileira de Zootecnia, v.30, p.1984-1988, 2001. (Suplemento).

FERRAZ FILHO, P.B.; RAMOS, A. de A.; SILVA, L.O.C. da; SOUZA, J.C. de; ALENCAR, M.M. de; MALHADO, C.H.M. Tendência genética dos efeitos direto e materno sobre os pesos à desmama e pós-desmama de bovinos da raça Tabapuã no Brasil. Revista Brasileira de Zootecnia, v.31, p.635-640, 2002.

FRANKHAM, R. Conservation genetics. Annual Review of Genetics, v.29, p.305-327, 1995.

GIANNOTTI, J. di G.; PACKER, I.U.; MERCADANTE, M.E.Z. Meta-análise para as estimativas de herdabilidade para características de crescimento em bovinos de corte. Revista Brasileira de Zootecnia, v.34, p.1173-1180, 2005.

GODDARD, M.G.; SMITH, C. Optimum number of bull sires in dairy cattle breeding. Journal of Dairy Science, v.73, p.11131122, 1990.

GUTIÉRREZ, J.P.; GOYACHE, F. A note on ENDOG: a computer program for analysing pedigree information. Journal of Animal Breeding and Genetics, v.122, p.172-176, 2005. 
LIRA, T. de; ROSA, E.M.; GARNERO, A. del V. Parâmetros genéticos de características produtivas e reprodutivas em zebuínos de corte (revisão). Ciência Animal Brasileira, v.9, p.1-22, 2008.

LÔBO, R.N.B.; MADALENA, F.E.; VIEIRA, A.R. Average estimates of genetic parameters for beef and dairy cattle in tropical regions. Animal Breeding Abstracts, v.68, p.433-462, 2000.

MALHADO, C.H.M.; MARTINS FILHO, R.; LÔBO, R.N.B.; FACÓ, O.; AZEVEDO, D.M.M.R.; SOUZA, J.C. de; OLIVEIRA, S.M.P. Tendência genética para características relacionadas à velocidade de crescimento de bovinos Nelore na região Nordeste do Brasil. Revista Brasileira de Zootecnia, v.34, p.60-65, 2005.

MALHADO, C.H.M.; RAMOS, A. de A.; CARNEIRO, P.L.S.; AZEVEDO, D.M.M.R.; MARTINS FILHO, R.; SOUZA, J.C. de. Melhoramento e estrutura populacional de bubalinos da raça Mediterrâneo no Brasil. Pesquisa Agropecuária Brasileira, v.43, p.215-220, 2008.

MEUWISSEN, T.H.E.; WOOLLIAMS, J. Effective sizes of livestock populations to prevent a decline in fitness. Theoretical and Applied Genetics, v.89, p.1019-1026, 1994.

MUIR, W.M. The interaction of selection intensity, inbreeding depression, and random genetic drift on short and long-term response to selection: results using finite locus and finite population size models incorporating directional dominance. Journal of Animal Science, v.79, p.1-11, 2000.

OLIVEIRA, J.A. de; LÔBO, R.B.; NUNES, H.O de. Tendência genética em pesos e ganhos de pesos em bovinos da raça Guzerá. Pesquisa Agropecuária Brasileira, v.30, p.1355-1360, 1995.

SANTOS, P.F.; MALHADO, C.H.M.; CARNEIRO, P.L.S.; MARTINS FILHO, R.; AZEVEDO, D.M.M.R.; MACHADO, C.H. Tendência genética, fenotípica e ambiental para o peso ao desmame de bovinos da raça Indubrasil no Estado da Bahia. Revista Científica de Produção Animal, v.9, p.18-24, 2007.

SAS INSTITUTE. SAS: user guide: versão 8. Cary: SAS Institute, 1999.

VALERA, M.; MOLINA, A.; GUTIÉRREZ, J.P.; GÓMEZ, J.; GOYACHE, F. Pedigree analysis in the Andalusian horse: population structure, genetic variability and influence of the Carthusian strain. Livestock Production Science, v.95, p.57-66, 2005.

VERCESI FILHO, A.E.; FARIA, F.J.C.; MADALENA, F.E.; JOSAHKIAN, L.A. Estrutura populacional do rebanho Tabapuã registrado no Brasil. Arquivo Brasileiro de Medicina Veterinária e Zootecnia, v.54, p.609-617, 2002. 